\title{
The Dissolution Dilemma for Low Pt Loading Polymer Electrolyte Membrane Fuel Cell Catalysts
}

Sandbeck, Daniel J.S.; Secher, Niklas Morch; Inaba, Masanori; Quinson, Jonathan; Sorensen, Jakob Ejler; Kibsgaard, Jakob; Zana, Alessandro; Bizzotto, Francesco; Speck, Florian D.; Paul, Michael T.Y. Total number of authors:

16

Published in:

Journal of the Electrochemical Society

Link to article, DOI:

10.1149/1945-7111/abc767

Publication date:

2020

Document Version

Publisher's PDF, also known as Version of record

Link back to DTU Orbit

Citation $(A P A)$ :

Sandbeck, D. J. S., Secher, N. M., Inaba, M., Quinson, J., Sorensen, J. E., Kibsgaard, J., Zana, A., Bizzotto, F., Speck, F. D., Paul, M. T. Y., Dworzak, A., Dosche, C., Oezaslan, M., Chorkendorff, I., Arenz, M., \& Cherevko, S. (2020). The Dissolution Dilemma for Low Pt Loading Polymer Electrolyte Membrane Fuel Cell Catalysts. Journal of the Electrochemical Society, 167(16), [164501]. https://doi.org/10.1149/1945-7111/abc767

\section{General rights}

Copyright and moral rights for the publications made accessible in the public portal are retained by the authors and/or other copyright owners and it is a condition of accessing publications that users recognise and abide by the legal requirements associated with these rights.

- Users may download and print one copy of any publication from the public portal for the purpose of private study or research.

- You may not further distribute the material or use it for any profit-making activity or commercial gain

- You may freely distribute the URL identifying the publication in the public portal 
OPEN ACCESS

The Dissolution Dilemma for Low Pt Loading Polymer Electrolyte Membrane Fuel Cell Catalysts

To cite this article: Daniel J. S. Sandbeck et al 2020 J. Electrochem. Soc. 167164501

View the article online for updates and enhancements. 


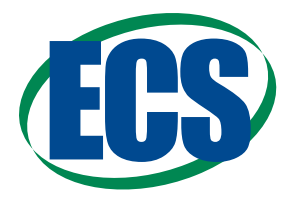

\title{
The Dissolution Dilemma for Low Pt Loading Polymer Electrolyte Membrane Fuel Cell Catalysts
}

\author{
Daniel J. S. Sandbeck, ${ }^{1,2,=, z}$ (i) Niklas Mørch Secher, ${ }^{3,=}$ (i) Masanori Inaba, ${ }^{4,5}$ (i) \\ Jonathan Quinson, ${ }^{4}$ (i) Jakob Ejler Sørensen, ${ }^{3}$ (i) Jakob Kibsgaard, ${ }^{3}$ (i) Alessandro Zana, ${ }^{6}$ (i) \\ Francesco Bizzotto, ${ }^{6}$ () Florian D. Speck, ${ }^{1,2}$ (1) Michael T. Y. Paul, ${ }^{1}$ (i) Alexandra Dworzak, ${ }^{7,8}$ (D) \\ Carsten Dosche,,$^{7,8}$ Mehtap Oezaslan, ${ }^{7,8, *}$ (i) Ib Chorkendorff, ${ }^{3}$ (i) Matthias Arenz, ${ }^{4,6, z} \mathbb{C}$ and \\ Serhiy Cherevko ${ }^{1, z}$ (i)
}

\author{
${ }^{1}$ Helmholtz-Institute Erlangen-Nürnberg for Renewable Energy (IEK-11), Forschungszentrum Jülich GmbH, 91058 \\ Erlangen, Germany \\ ${ }^{2}$ Department of Chemical and Biological Engineering, Friedrich-Alexander-Universität Erlangen-Nürnberg, 91058 \\ Erlangen, Germany \\ ${ }^{3}$ Department of Physics, Technical University of Denmark, 2800 Lyngby, Denmark \\ ${ }^{4}$ Department of Chemistry, University of Copenhagen, DK-2100 Copenhagen $\emptyset$, Denmark \\ ${ }^{5}$ Toyota Central R\&D Labs, Inc., Nagakute, Aichi 480-1192, Japan \\ ${ }^{6}$ Department of Chemistry and Biochemistry, University of Bern, CH-3012 Bern, Switzerland \\ ${ }^{7}$ Department of Chemistry, Carl von Ossietzky University of Oldenburg, 26111 Oldenburg, Germany \\ ${ }^{8}$ Institute of Technical Chemistry, Technical University of Braunschweig, 38106 Braunschweig, Germany
}

Cost and lifetime currently hinder widespread commercialization of polymer electrolyte membrane fuel cells (PEMFCs). Reduced electrode Pt loadings lower costs; however, the impact of metal loading (on the support) and its relation to degradation (lifetime) remain unclear. The limited research on these parameters stems from synthetic difficulties and lack of in situ analytics. This study addresses these challenges by synthesizing 2D and 3D Pt/C model catalyst systems via two precise routes and systematically varying the loading. Pt dissolution was monitored using on-line inductively coupled plasma mass spectrometry (on-line-ICP-MS), while X-ray spectroscopy techniques were applied to establish the oxidation states of $\mathrm{Pt}$ in correlation with metal loading. Dissolution trends emerge which can be explained by three particle proximity dependent mechanisms: (1) shifts in the Nernst dissolution potential, (2) redeposition, and (3) alteration of Pt oxidation states. These results identify engineering limitations, which should be considered by researchers in fuel cell development and related fields.

(C) 2020 The Author(s). Published on behalf of The Electrochemical Society by IOP Publishing Limited. This is an open access article distributed under the terms of the Creative Commons Attribution Non-Commercial No Derivatives 4.0 License (CC BYNC-ND, http://creativecommons.org/licenses/by-nc-nd/4.0/), which permits non-commercial reuse, distribution, and reproduction in any medium, provided the original work is not changed in any way and is properly cited. For permission for commercial reuse, please email: permissions@ioppublishing.org. [DOI: 10.1149/1945-7111/abc767]

\begin{abstract}
Manuscript submitted September 2, 2020; revised manuscript received October 23, 2020. Published November 20, 2020. This paper is part of the JES Focus Issue on Proton Exchange Membrane Fuel Cell and Proton Exchange Membrane Water Electrolyzer Durability.
\end{abstract}

Supplementary material for this article is available online

For years' polymer electrolyte membrane fuel cell (PEMFC) electrocatalysis research has been aimed at increasing catalyst activity towards the sluggish oxygen reduction reaction (ORR), in an effort to decrease the total Pt loading necessary in the cathode catalyst layer. By decreasing Pt loading the total $\operatorname{cost}^{1}$ and amount of $\mathrm{Pt}$ in circulation can be reduced, thereby increasing manufacturing production volumes of fuel cell electric vehicles. $^{2}$

Several strategies have been suggested to increase catalyst activity, with major research efforts focused on Pt alloys and shape-controlled Pt nanoparticles. ${ }^{3-10}$ Although such advanced materials show promising ORR activity, less can be said regarding their stability. Furthermore, on the pathway to lower Pt loadings, it is of immediate interest to understand the degradation behavior of current state-of-the-art materials. ${ }^{11}$

Decreased Pt loading achieved via thin catalyst layers is known to severely compromise performance at high current densities in membrane electrode assemblies (MEAs). ${ }^{1,2}$ Such loading dependent performance has been attributed to flooding and a local $\mathrm{O}_{2}$ resistance related to changing $\mathrm{Pt}$ substrate-ionomer interactions when going from thick to thin ionomer films. In contrast, the mechanisms behind the observed increase in irreversible degradation can only be speculated without dedicated investigations.

\footnotetext{
$=$ These authors contributed equally to this work.

* Electrochemical Society Member.

żE-mail: d.sandbeck@fz-juelich.de; matthias.arenz@dcb.unibe.ch; s.cherevko@fzjuelich.de
}

Under well-defined half-cell conditions, which become necessary for elucidating fundamental phenomena undetectable in complex systems, studying the impact of catalyst layer thickness on the primary degradation mechanism of Pt dissolution revealed crucial phenomena. ${ }^{12,13} \mathrm{On}$ line dissolution measurements with a scanning flow cell coupled to an inductively coupled plasma mass spectrometer (SFC-ICP-MS) showed that electrochemical specific dissolution normalized by electrochemically active surface area (ECSA) increased with decreasing catalyst layer thickness. While the exact mechanism remains unknown, this observed dissolution trend was tentatively attributed to a shift in the Nernst potential for dissolution, which was greater for the increased diffusional path lengths of thicker catalyst layers. Due to these unambiguous performance and degradation issues, lower Pt content via catalyst layer thickness will be limited to a yet to be defined length without further materials improvements.

Considering the perceived limitations of thin catalyst layers, a new contrasting approach to lower Pt loading must be investigated. By lowering the Pt density within the catalyst layer, i.e. the wt.\% Pt, the Pt content can be varied independent of thickness. Similar to the low Pt content of thin catalyst layers, low Pt density has been reported to decrease ORR activity. ${ }^{14-17}$ However, in comparison to catalyst layer thickness, the dependence of Pt density on degradation remains largely unexplored. ${ }^{15,16,18,19}$ Therefore, in this work the SFC-ICP-MS technique was employed to elucidate the effect of loading on dissolution with constant catalyst layer thickness. Threedimensional (3D) Pt/Vulcan materials and two-dimensional (2D) model magnetron-sputtered mass-selected systems of varied loadings were investigated. For the $3 \mathrm{D} \mathrm{Pt} / \mathrm{C}$ materials, exclusive control of material properties such as particle size and loading was achieved 
with a two-step surfactant-free polyol synthetic technique, termed the "toolbox" approach. ${ }^{14,15,20-24}$ Moving to the 2D model systems, particle size and distribution were precisely tuned using a magnetron-based cluster source where Pt clusters were deposited on a planar glassy carbon substrate. ${ }^{17}$ By studying model 2D systems, further analytical confirmation becomes possible which ensures firm conclusions on the complex, applied 3D systems. For direct comparison, the common parameter of the edge-to-edge interparticle distance (ipd) is used, which was recently shown to be crucial in the degradation of $\mathrm{Pd}$ heterogeneous catalysts ${ }^{25}$ and now also to electrocatalyst degradation via Pt dissolution.

\section{Results}

By studying well-defined model 2D (mass-selected, magnetron sputtered) in parallel to the applied 3D (Pt/Vulcan) materials, the combined simplicity of the former and complexity of the latter reaffirm the conclusions to be discussed. The model 2D materials eliminate possible support effects, contaminations from wet-chemical syntheses and allow precise calculation of interparticle distance, ${ }^{13,20,21,26}$ while the 3D Pt/Vulcan materials provide a realistic applied material. The critical parameters of particle size and interparticle distance for both the $2 \mathrm{D}$ and $3 \mathrm{D}$ materials were analyzed and are shown in Fig. 1. SEM images of the 1, 2 and 5\% coverages for the 2D materials are displayed in the top row of Figs. 1a-1c. At higher coverages, the interparticle distances become difficult to estimate from the SEM images due to poor resolution and increasing probability of particle overlap; therefore, the three lowest coverages are used to estimate values of interparticle distance from the SEM images and are plotted in Fig. 1d. To obtain interparticle distances at higher coverages, it is possible to use calculations assuming a homogenous (evenly spaced) distribution. However, it is seen in Fig. 1d (magnified in Fig. S1 is available online at stacks.iop. org/JES/167/164501/mmedia) that this would overestimate the interparticle distances at low coverages. Therefore, the interparticle distances were simulated by generating random particle positions with the number of particles in a given area determined by coverage (detailed in supplementary information), which fit well with the measured SEM values and are used in the following discussion. To ensure a narrow size distribution as a result from the synthesis, Pt particles were deposited on a $\mathrm{Cu}$ TEM grid and analyzed via STEM. The resulting particle size distribution is displayed in Fig. 1e (full image sets are found in Fig. S4). Two narrow distributions were obtained, the single mass and the double mass particles. Due to the low number of double mass particles these can be neglected. A mean diameter of $6.5 \pm 0.4 \mathrm{~nm}$ was obtained. The deviation from 6 to $6.5 \mathrm{~nm}$ is due to the error of the assumption of spherical particles when calculating particle size from mass. The particles are not perfect spheres as is evident from Fig. S4b. A narrow distribution with mean diameter of $6.8 \pm 0.7 \mathrm{~nm}$ was obtained, with a small fraction of larger particles which unavoidably result from doubly charged particles passing through the mass filter. The use of rather large particle sizes here enabled the estimation of interparticle distances at low coverages confirming the accuracy of the simulation and enabling it predict values at higher coverages, which becomes nearly impossible for smaller particle sizes due to the limited resolution of SEM analysis.

Analysis of the 3D Pt/Vulcan materials was carried out using TEM, and is analogously displayed with increasing loading in the bottom row of Figs. 1f-1h (a full set of images is found in Fig. S5). Highly homogeneous particle distributions and increasing loading of NPs are clearly observed. In contrast to the 2D materials, estimation of the interparticle distance becomes difficult from a 2D image of a 3D material, ${ }^{15,22}$ highlighting the increased complexity of moving from model to applied catalyst systems. Therefore, for the 3D systems, the interparticle distance was calculated using Eq. S1 which assumes homogeneously spaced particles and is shown in Fig. 1i (magnified in Fig. S3) for all Pt/Vulcan materials. However, it should be noted that for the $2 \mathrm{D}$ materials this treatment overestimates the distances at low coverages, as similarly reported for $\mathrm{Pt} /$ Vulcan previously. ${ }^{22}$ The particle size distribution of each material was measured and a representative histogram is displayed in Fig. 1j (a full set of data is found in Fig. S6). The particle sizes were found to be identical within error (1.9 $\pm 0.5 \mathrm{~nm}$ average), as to be expected with the two-step synthetic approach which yields high control over particle size and loading. Further analysis on the crystalline nature of such synthesized Pt nanoparticles was carried out previously by Quinson et al. ${ }^{20}$

Aside from the physical properties on the nanoscale of the material, macroscopic properties are critical in fundamental electrochemical studies. The 2D materials are sputtered in a raster pattern (Fig. S7) over a large area of glassy carbon, relative to the contact area of the SFC comprising the working electrode. Therefore, the $\mathrm{Pt}$ coverage must be highly homogeneous to ensure that any area can be contacted and measured electrochemically. By measuring an array of XPS spectra and quantifying the atomic content of Pt across each sample (Figs. S8 and S9) the homogeneity of each sample was
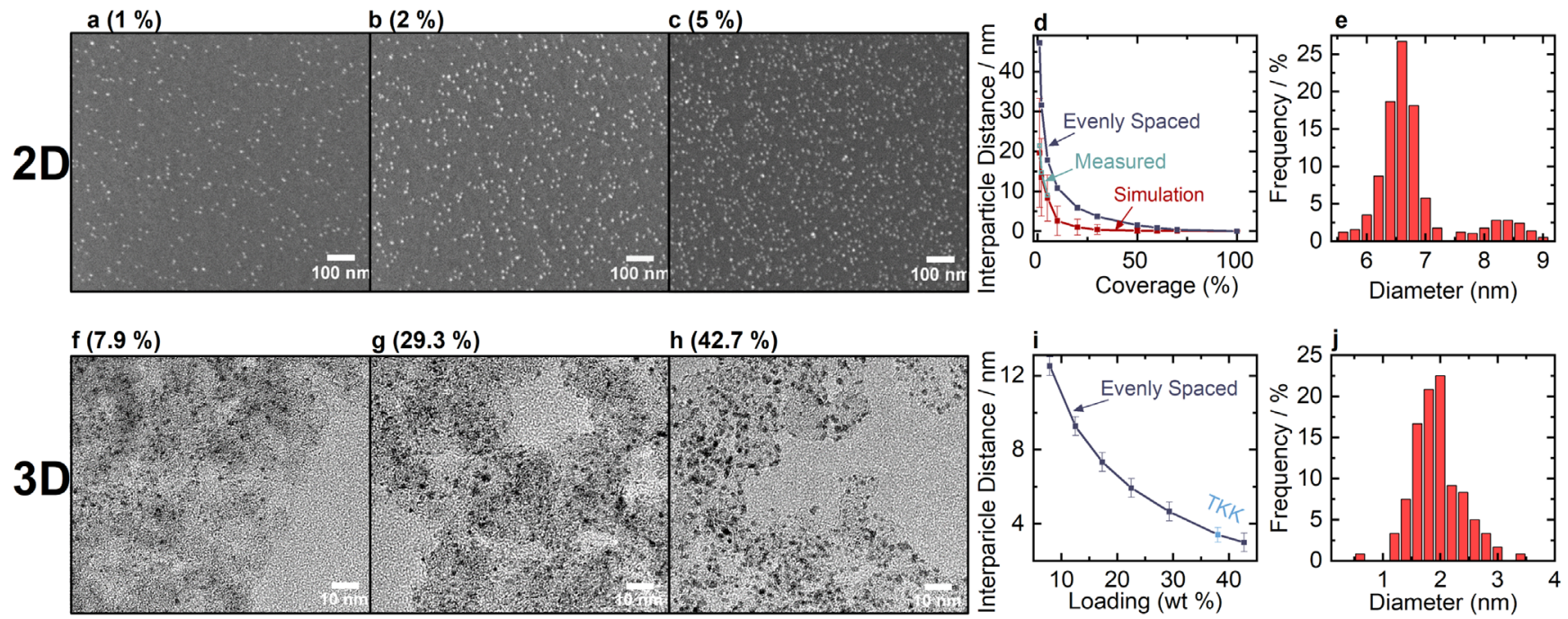

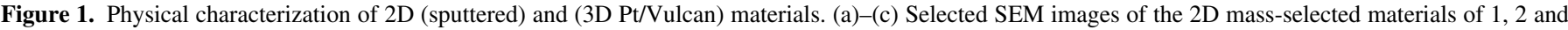

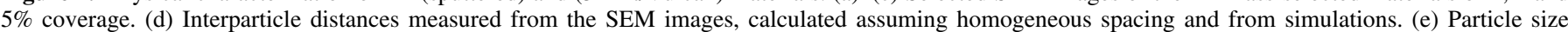

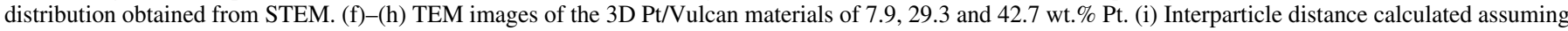

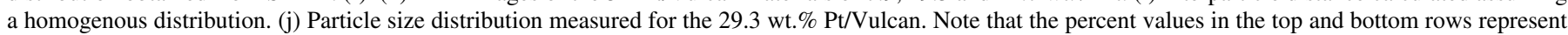
$\mathrm{Pt}$ coverage and $\mathrm{Pt}$ to carbon mass ratio, respectively. 

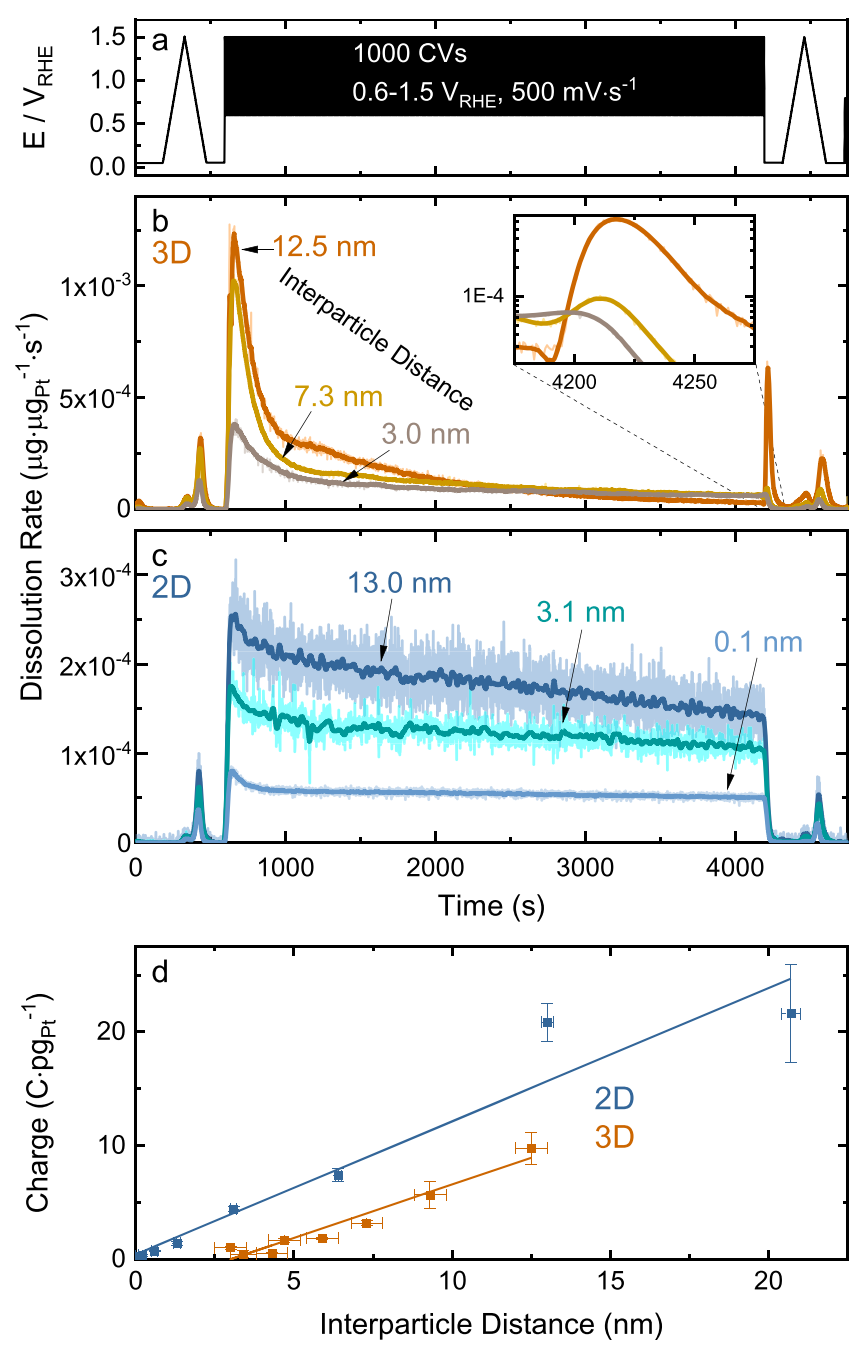

Figure 2. Pt dissolution during the ADT. (a) Full ADT protocol of CVs from 0.6-1.5 $\mathrm{V}_{\mathrm{RHE}}, 500 \mathrm{mV} \cdot \mathrm{s}^{-1}$ for 1000 cycles. (b) Resultant dissolution rate profiles for the 3D Pt/Vulcan and (c) 2D magnetron-sputtered systems for three interparticle distances. (d) The mass-normalized charge during the $0.05 \mathrm{~V}_{\text {RHE }}$ reductive hold immediately following the ADT cycling $(\mathrm{n} \geqslant 3)$.

assured after synthesis. For the $70 \%$ coverage sample, a relative standard deviation of only $3.4 \%$ was obtained, demonstrating the effectiveness of the raster technique. In addition to ensuring coverage homogeneity, the XPS spectra (Fig. S8) indicate a clean surface with no contaminations while the atomic content of Pt shows a linear increase with coverage (Fig. S10), indicating that significant particle overlap is only taking place at coverages $>50 \%$.

In contrast to the $2 \mathrm{D}$ materials sputtered over a large piece of glassy carbon, the 3D Pt/Vulcan materials are dropcast as spots onto glassy carbon, and completely enclosed by the SFC contact area. The amount of Pt on the working electrode will be determined simply by the volume of ink dropcast; however, constant and uniform catalyst layer thickness becomes a prerequisite to separate the previously reported effects of varying thickness from $\mathrm{Pt}$ density (i.e. wt.\% loading). Therefore, profilometry was employed as a screening tool. A constant average thickness/height of $0.4 \mu \mathrm{m}$ was found for all samples as shown in Fig. S11. Microscopy and profilometry (Figs. S12 and S13) show homogenous catalyst layer surfaces with no "coffee ring"22,27-33 effect and only slight agglomeration in the middle of the spots. However, the commercial Pt/Vulcan (TKK) catalyst has a significant amount of agglomeration and some degree of the coffee ring effect, causing a bumpy surface. The differing dropcasting results could possibly be due to varying properties of the Vulcan support, such as surface area and/or functionalization degree.
It is well known that small variations of parameters can yield vastly different dropcasting results. ${ }^{22,27,29-32,34}$

To measure the electrochemical dissolution of the 2D and 3D systems the samples were subjected to a cyclic voltammogram (CV, $\left.0.05-1.5 \mathrm{~V}_{\mathrm{RHE}}\right)$ with a slow scan rate $\left(10 \mathrm{mV} \cdot \mathrm{s}^{-1}\right)$ followed by aggressive accelerated degradation test (ADT) protocol (1000 cycles of $0.6-1.5 \mathrm{~V}_{\mathrm{RHE}}, 500 \mathrm{mV} \cdot \mathrm{s}^{-1}$ ) and finally another "slow" $\mathrm{CV}$, as seen in Fig. 2a. By employing these two specific scan rates and potential windows, different aspects of dissolution are elucidated. The potential window used here has been demonstrated to cause significant Pt dissolution with rather minimal carbon corrosion. ${ }^{35}$ In comparison, the typically used potential windows in accelerated degradation tests in the range of 0.6-1.0 $\mathrm{V}_{\mathrm{RHE}}$ and 1.0-1.5 $\mathrm{V}_{\mathrm{RHE}}$ (used to simulate load and start/stop conditions, respectively), will not cause significant $\mathrm{Pt}$ dissolution in such a half-cell experiment designed for online detection. The impact of potential window will additionally be addressed in an upcoming manuscript.

The mass-normalized dissolution rate profiles for the $3 \mathrm{D}$ and $2 \mathrm{D}$ systems during the ADT are shown in Figs. $2 b$ and $2 c$, which caused extensive Pt dissolution from 15\%-65\% loss for the 2D and 3D systems (Fig. S14). No particle detachment is observed in the ICPMS signal (which would cause excessively large spikes), even in the absence of ionomer, indicating strong binding of the nanoparticles to oxygenated support sites, as observed previously for the Pt/Vulcan toolbox synthesis method. ${ }^{21}$ Dissolution clearly decreases with decreasing interparticle distance (increasing loading), but here the distinct shapes of the profiles are strikingly different. For the 3D systems, the rate drastically decreases in the initial stages of the ADT, which is far more pronounced for large interparticle distances. At the end of the ADT, an additional cathodic dissolution peak is observed only at large interparticle distances when a reductive potential of $0.05 \mathrm{~V}_{\text {RHE }}$ was applied (Fig. 2b, inset). This is most pronounced for the $12.5 \mathrm{~nm}$ interparticle distance and is only observed for the three largest interparticle distances of the 3D systems. Such behavior is absent from the rather linear decline in dissolution rate of the 2D system, which has larger $6.8 \mathrm{~nm}$ particles in comparison to $1.9 \mathrm{~nm}$ for $\mathrm{Pt} / \mathrm{Vulcan}$.

It appears that for large interparticle distances, the Pt nanoparticles become (partially) passivated by formed $\mathrm{PtO}_{\mathrm{x}}$, i.e. the lower potential limit (LPL) of $0.6 \mathrm{~V}_{\mathrm{RHE}}$ is too positive to fully reduce this formed layer on the time scale of the ADT cycling. This is further illustrated in the mass-normalized charge during the reductive hold following the ADT in Fig. 2d (error from $n \geqslant 3$ where raw data treatment is shown in Figs. S15 and S16). The mass-normalized charge is used to take into account variations of Pt mass on the glassy carbon substrate. The larger interparticle distances contain more $\mathrm{PtO}_{\mathrm{x}}$ at the end of the ADT and thus more charge is passed to the nanoparticles, which leads to a higher accumulated charge measured during the reductive hold. This is further pronounced by the Gibbs-Thomson effect ${ }^{36-39}$ for the 3D system of smaller particle size, causing the additional cathodic peak at the end of the ADT.

The intercept of the mass-specific reduction charge (Fig. 2d) is shifted for the 3D materials, which theoretically must go through the origin (as interparticle distance approaches zero the relative Pt mass approaches infinite values). Calculation of interparticle distances for the 3D materials assumed homogeneously distributed, evenly spaced particles, due to lack of accurate experimental techniques. For the 2D materials this was shown by SEM and simulations to overestimate interparticle distances (Fig. 1d), while for Pt/Vulcan it has been suggested that inaccessible pores cause an overestimation of surface area (and thus interparticle distance), which explains the observed shift of the intercept away from the origin. This result highlights the importance of comparing well-defined model systems to applied materials on the quest to understand electrocatalytic materials and their degradation.

To further explore the phenomena of particle passivation, which may be compounded by particle size shrinking during the ADT (due to dissolution), an "extended" ADT to 2500 cycles was applied to the $20.7 \mathrm{~nm}$ interparticle distance (1\% coverage) 2D sample. As 

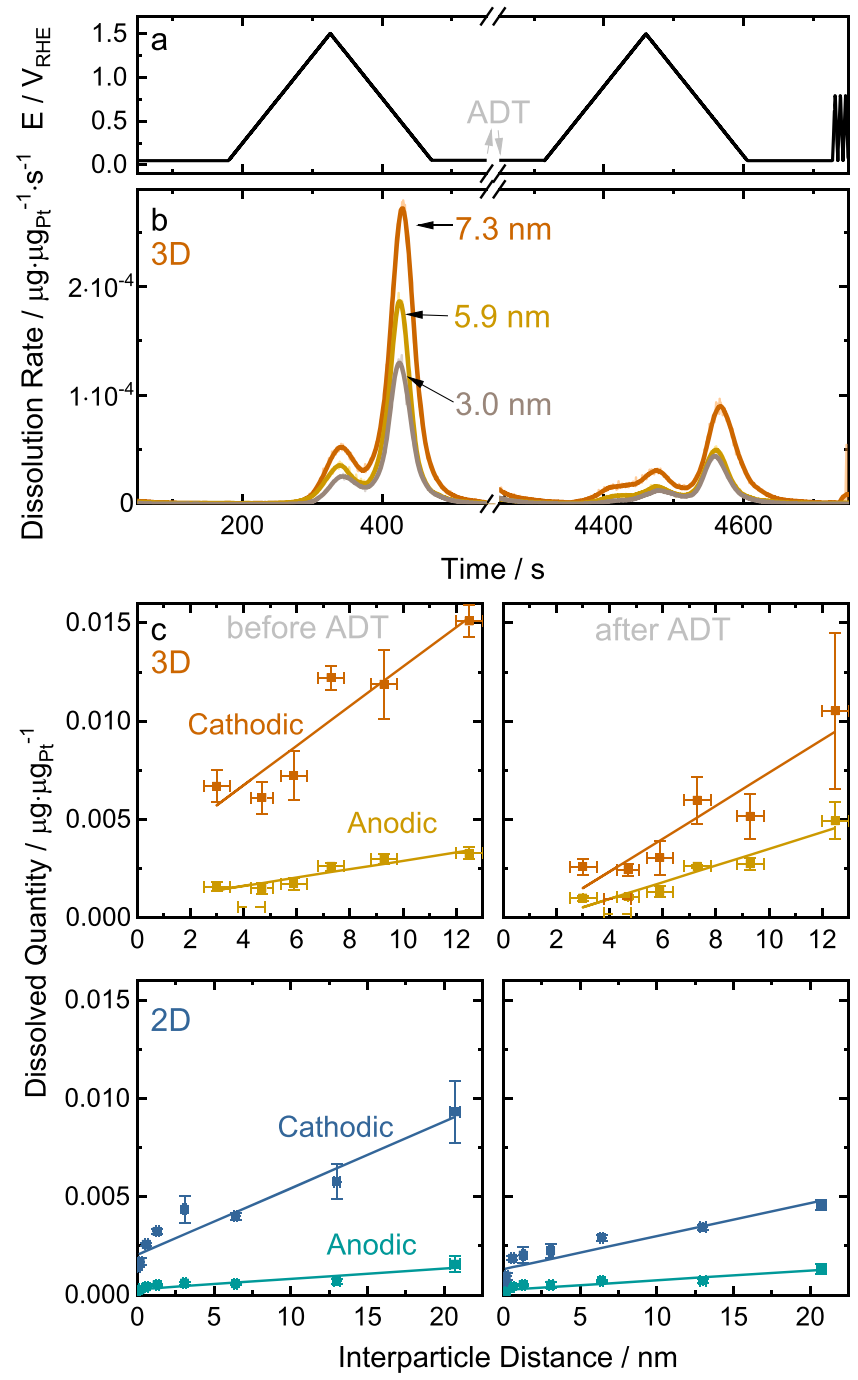

Figure 3. Pt dissolution preceding and following the ADT. (a) Two CVs from $0.05-1.5 \mathrm{~V}_{\mathrm{RHE}}$ at $10 \mathrm{mV} \cdot \mathrm{s}^{-1}$ prior and following a harsh ADT protocol from $0.6-1.5 \mathrm{~V}_{\mathrm{RHE}}, 500 \mathrm{mV} \cdot \mathrm{s}^{-1}$ for 1000 cycles. (b) Resultant dissolution rate profiles normalized to total Pt mass on the glassy carbon substrate for varying interparticle distance. (c) Quantified anodic and cathodic dissolution before and after the ADT normalized to mass for the 3D Pt/Vulcan (top) and $2 \mathrm{D}$ magnetron-sputtered (bottom) materials $(\mathrm{n} \geqslant 3)$.

shown in Fig. S17, $280 \%$ of the initial material was lost as the dissolution rate decreases to near baseline values, indicating highly passivated Pt particles. In comparison $\sim 60 \%$ Pt loss was observed in Fig. S14 for 1000 cycles for the same material. After 2500 cycles the potential was held again at $0.05 \mathrm{~V}_{\mathrm{RHE}}$ and a large spike in the dissolution rate was observed, due to the reduction of oxidepassivated Pt particles. The reduction charge after 2500 cycles of ADT was found to be identical to after 1000 cycles, which follows that the dissolution rate is approaching low values and the particles are approaching full passivation by this point of the experiment.

The slower CVs preceding and following the ADT have a larger potential window with a more negative lower potential limit (LPL) of $0.05 \mathrm{~V}_{\mathrm{RHE}}$, in comparison to $0.6 \mathrm{~V}_{\mathrm{RHE}}$ during the ADT. Therefore, the formed oxide layers should be fully reduced during the cathodic scan down to $0.05 \mathrm{~V}_{\mathrm{RHE}}$ and eliminate formed passivating layers skewing results, thus the intrinsic interparticle distance effect on Pt dissolution can be observed. Figure $3 \mathrm{~b}$ shows the electrochemical mass-normalized $\mathrm{Pt}$ dissolution rate profiles for the 3D systems during the two slow CVs before and after the ADT. The separate anodic and cathodic dissolution processes are visible at this relatively slow scan rate (unobservable during the fast ADT

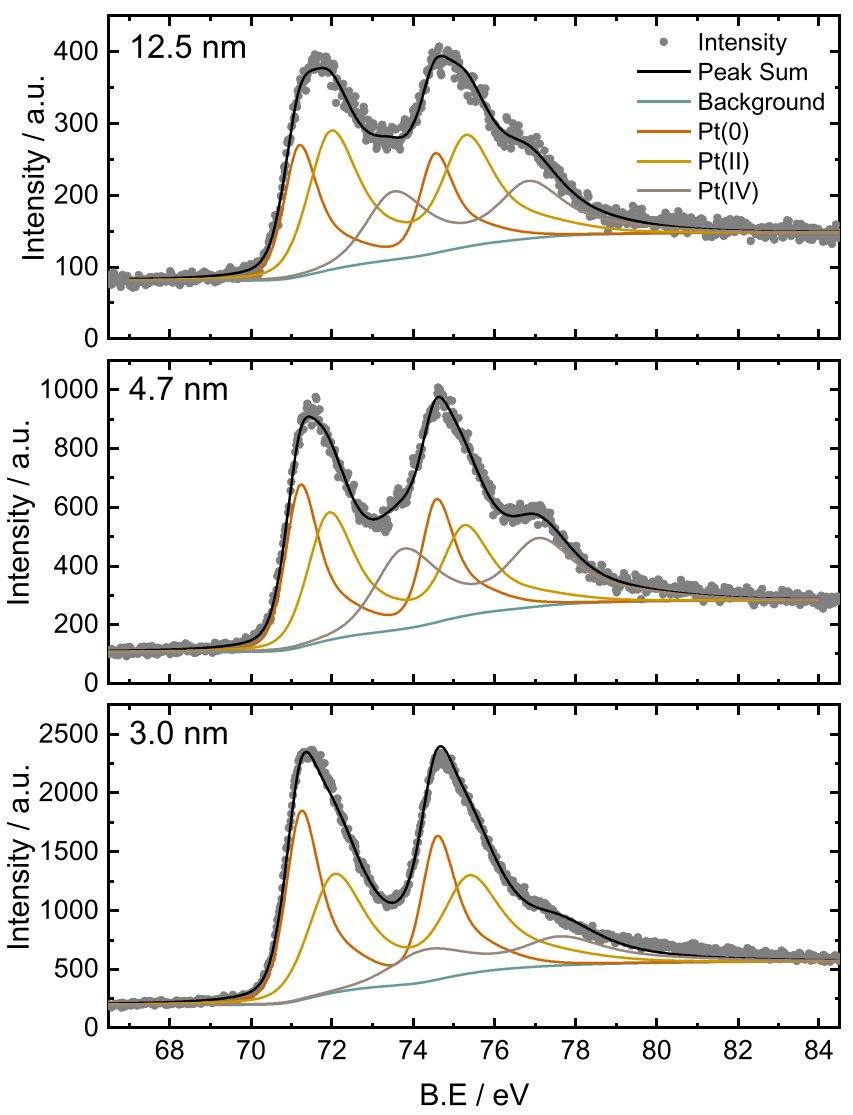

Figure 4. Ex-situ Pt 4f XPS spectra of the 12.5, 4.7 and $3.0 \mathrm{~nm}$ interparticle distance samples, showing the measured intensity and fitted spectra.

scans) and dissolution is clearly diminished after the ADT (expected after significant Pt loss). Some degree of tailing is seen, as compared to slower $2 \mathrm{mV} \cdot \mathrm{s}^{-1}$ scans. ${ }^{13}$ Most importantly, a trend is observed for both 2D and 3D systems in which dissolution decreases with decreasing interparticle distance, exemplified in the dissolved quantities for the anodic and cathodic peaks in Fig. 3c. A similar observation for varying catalyst layer thickness and constant interparticle distance was earlier attributed to a diffusional effect causing a shift in the Nernst potential for dissolution. ${ }^{13}$ An additional observation is seen in the anodic dissolution peak after the ADT (only for Pt/Vulcan materials), where a shoulder appears with an onset $\leqslant 0.5 \mathrm{~V}_{\mathrm{RHE}}$. Dissolution during the ADT will cause the shrinking of some particles to sub-nanometer sizes which may be susceptible to dissolution at such lower potentials. ${ }^{13,40,41}$ Further ILTEM experiments before and after the ADT on the 3D Pt/Vulcan material indeed show particle shrinking caused by dissolution (Fig. S18), and that dissolution rather than agglomeration appears to the primary degradation mechanism during this ADT experiment.

Ex-situ XPS, XANES and EXAFS characterization techniques were used to further corroborate the above observations of increasing nanoparticle oxidation at larger interparticle distances for the as-prepared 3D Pt/Vulcan materials. Considering that the 2D mass-selected materials are limited to flat surfaces for accurate control of interparticle distance and that the 3D Pt/Vulcan materials with smaller particle sizes showed a much more drastic passivation effect on the Pt dissolution above, only the 3D applied systems were characterized further. By analyzing the $\mathrm{Pt} 4 \mathrm{f}$ XPS peak positions and areas it is possible to ascertain the relative amount of Pt surface oxidation. The fitted XPS spectra of 3.0, 4.7 and $12.5 \mathrm{~nm}$ interparticle distance Pt/Vulcan materials are shown in Fig. 4 and as interparticle distance increases, the relative amounts of $\mathrm{Pt}(0)$ decreases and the Pt $4 \mathrm{f}$ peak shifts to higher binding energies (Table SII), indicating increased oxidation state of the Pt species. 


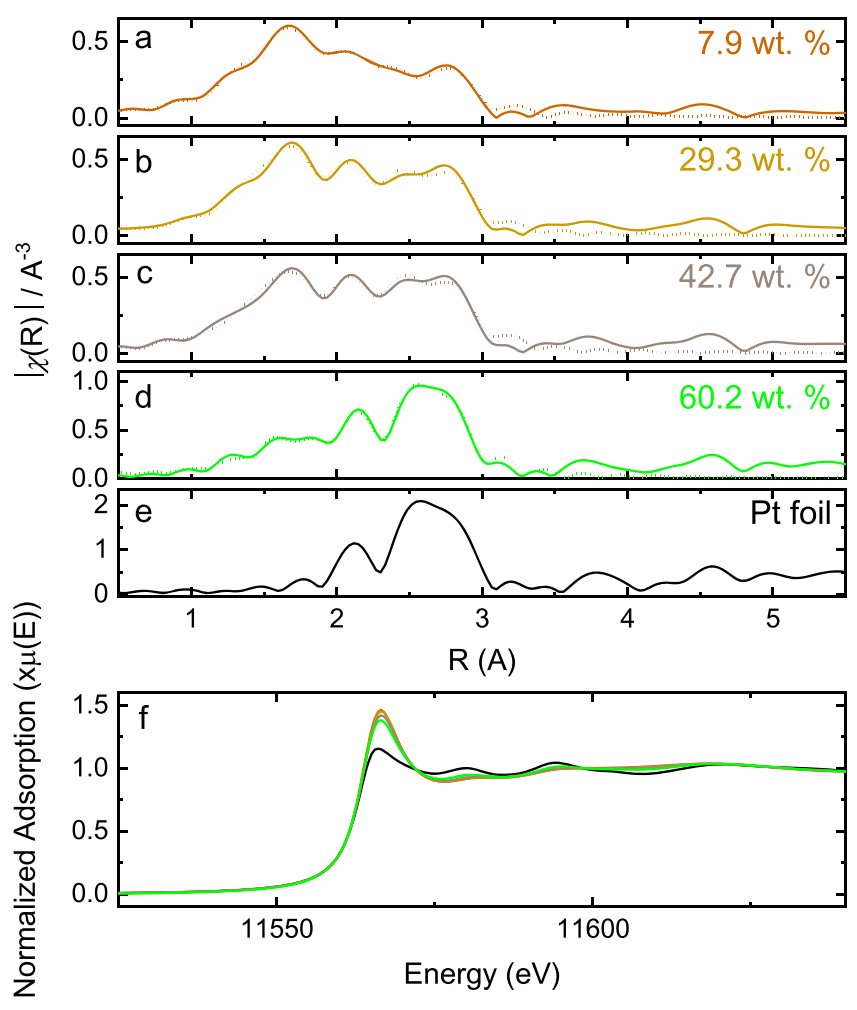

Figure 5. FT-transformed magnitudes of the $\mathrm{k}^{2}$-weighted EXAFS spectra and fits (dotted) for interparticle distances of (a) $7.9 \mathrm{wt} . \%$, (b) $29.3 \mathrm{wt} . \%$, (c) 42.7 wt.\%, (d) 60.2 wt.\% and (e) Pt foil as reference. (f) Corresponding $\mathrm{L}_{\text {III }}$ XANES spectra.

With EXAFS and XANES characterization, inferences regarding the nearest neighbor coordination and the density of unoccupied states may be made, providing further information on the oxidation of the $\mathrm{Pt}$ NPs. The FT-transformed magnitudes of the $\mathrm{k}^{2}$-weighted EXAFS data and the corresponding fits for Pt/Vulcan materials with different loadings (decreasing interparticle distance with increasing loading) are shown in Fig. 5a. For analysis, the nearest neighbor Pt-O and Pt-Pt contributions to the EXAFS data were determined and summarized in Table SIII. The total coordination number of Pt (sum of the partial coordination numbers of $\mathrm{N}(\mathrm{Pt}-\mathrm{Pt})$ and $\mathrm{N}(\mathrm{Pt}-\mathrm{O})$ are similar irrespective of the loading, indicating that the particle size of the Pt NPs are in the same range. More interestingly, the contribution of Pt-O increases with decreasing loading. Despite similar total coordination numbers, the partial coordination number of Pt-O increases from $1.6 \pm 0.3$ to $2.8 \pm 0.8$ for 60.2 and 7.9 wt.\% loadings, respectively. Evidently, the oxophilic properties of these Pt NPs are enhanced with increasing interparticle distance. The changes in the oxidation state of Pt NPs coincide with changes in the white line intensity from the Pt $\mathrm{L}_{\mathrm{III}}$ edge XANES data (Fig. 5b), which corresponds closely to the density of unoccupied states and thus affinity to $\mathrm{PtOH}$ formation.

\section{Discussion}

The trends of decreasing dissolution rate and quantities with decreasing interparticle distance may be explained by three fundamental mechanisms, visually illustrated in Fig. 6 for two (massselected) materials, where smaller interparticle distances are shown in the foreground. Here the red arrows indicate hinder dissolution of Pt atoms as opposed to the unhindered white arrows. These three mechanisms are further described:

1) As interparticle distance decreases, local $\mathrm{Pt}^{\mathrm{n}+}$ ion concentrations will more quickly build up in the vicinity of the $\mathrm{Pt}$ nanoparticles, causing a shift in the Nernst equilibrium potential for electrochemical dissolution. ${ }^{13}$
2) As interparticle distance decreases, the probability for $\mathrm{Pt}^{\mathrm{n}+}$ ion redeposition on adjacent $\mathrm{Pt}$ particles or particles within the diffusion path from inside the electrode pores to the bulk electrolyte during cathodic potentials will increase.

3) As interparticle distance decreases, the Pt NPs begin to impact each other's oxophilicity. In acidic electrolyte there is increasing overlap between the Pt nanoparticles' electrochemical double layer (EDL), which shifts the oxidation potential of the particles to more positive potentials. Therefore, the Pt nanoparticles become less oxidized during the electrochemical protocol, ${ }^{14-17,42}$ which results in decreased dissolution. Such an overlap in the EDL was predicted for interparticle distances smaller than the Debey length, ${ }^{17}$ i.e. for interparticle distances smaller than $2 \mathrm{~nm}$ for chosen conditions. As evidenced by ex situ XPS and XANES/ EXAFS, there is also an impact on the electronic configuration of the NPs, which move from one of isolated NPs towards one of NP ensembles with screened coulomb interaction and more metallic film like properties. ${ }^{43-46}$ According to the shift in the oxygen reduction potential from CVs (Fig. S19) ${ }^{14-17,42}$ and the diminished d-band vacancy from XANES at low interparticle distances, there are changes in the affinity of Pt nanoparticles for oxidation. Consequently, and similar to the impact of double layer overlap, the Pt nanoparticles with lower interparticle distance will become less oxidized during the electrochemical protocol which results in decreased dissolution.

Although all suggested mechanisms (1-3) must contribute to the trends in mass-specific dissolution shown in Figs. 2 and 3, they cannot be the only factors at play. If the dissolution trend were entirely a consequence of diffusion controlled mechanisms (1) and (2) a directly proportional change in dissolution with coverage or loading should be expected. Mechanism (3) would also contribute to a more drastic decrease in specific dissolution when combined with (1) and (2); however, the dissolution decreases more gradually than can be accounted for by these mechanisms alone. In other words, the total dissolution rates and quantities should more than double if the interparticle distance doubles. The dissolution of Pt is one of many complex interconnected degradation mechanisms, but one possible explanation here is that dissolution is also being controlled kinetically due to low-coordinated defect sites (i.e. edges, corners, adislands and adatoms) present on the nanoparticles surface. ${ }^{47}$

While mechanisms (1-2) are arguably intuitive and have shown to play a role in dissolution on varied diffusion path lengths (catalyst layer thicknesses) at constant interparticle distance, mechanism (3) has a very interesting effect on electrochemical dissolution. During the 0.6-1.5 $\mathrm{V}_{\text {RHE }}$ ADT (Fig. 2), for both the 2D and 3D systems the Pt particles become (partially) passivated by $\mathrm{PtO}_{\mathrm{x}}$, which clearly depends on the interparticle distance. From the shape of the 3D dissolution rate profile, the sharp initial decrease indicates such passivation is pronounced at large interparticle distances, further evidenced by the cathodic dissolution peak during the subsequent reductive hold. Small interparticle distances diminish this effect and the particles are more easily reduced (become less passivated during the ADT). Similarly, mechanism (3) has previously been attributed to observed increases in ORR activity with decreasing interparticle distance for 2D and 3D systems, as poisoning oxygenated species can block active catalytic sites. ${ }^{14-17}$ Inhibited oxide formation also explains the observed shift in the Pt CV reduction peak (Fig. S19). ${ }^{14-17,42}$

The XPS and XANES data demonstrate that the shift in the Pt $\mathrm{CV}$ reduction peak is at least partially due to mechanism (3), i.e. a change in electronic configuration of $\mathrm{Pt}$ with decreasing interparticle distance. As interparticle distance increases, the relative amounts of $\mathrm{Pt}(0)$ decrease and $\mathrm{Pt}(\mathrm{II})$ and $\mathrm{Pt}(\mathrm{IV})$ increase, and consequently the $\mathrm{Pt} 4 \mathrm{f}$ peak shifts to higher binding energies in the XPS spectra. From the EXAFS experiments an increase in Pt-O coordination is seen, while XANES results clearly show a decreased d-band vacancy at small interparticle distances, resulting in less affinity to $\mathrm{PtOH}$ formation. This suggests that the interface between the particles is influenced by coupling/dipole-dipole interactions between the 


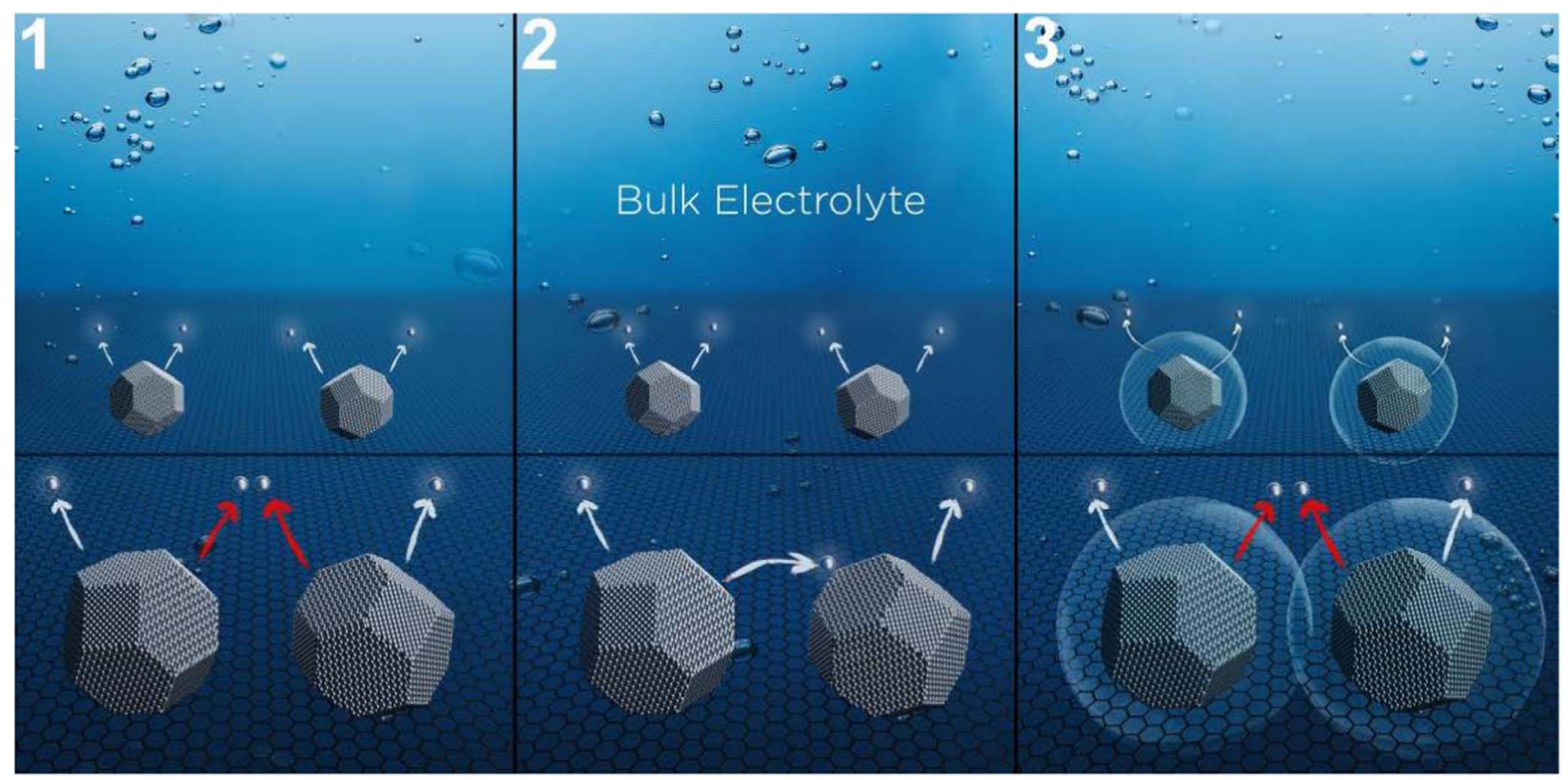

Figure 6. Schematic representation of mechanisms (1-3) on a planar carbon surfaces below a liquid electrolyte for (foreground) small interparticle distance and (background) large interparticle distance. White arrows show dissolved Pt atoms while red arrows indicate inhibited dissolution.

particles. Similar effects have been observed previously in the electronic structure of ensembles of metallic and semiconducting NPs, ${ }^{43-45}$ but so far dipole-dipole interactions have not been related to catalytic reactions. At interparticle distances between 0.5 and $10 \mathrm{~nm}$, dipole-dipole interactions can lead to energy transfer between neighboring nanoparticles. ${ }^{43}$ The length scale is well within the range of interparticle distances reported here.

Such coupling in closely-spaced NP systems has been shown to modify electronic structures and transport properties. In isolated sub$10 \mathrm{~nm}$ NPs the electronic structure can be described by isolated wavefunctions, but when brought in close proximity the wave functions can couple, forming bands transition from insulator to conductor with decreasing interparticle distance. ${ }^{43-46}$ Although the individual contributions of the double layer and electronic structure of mechanism (3) on observed dissolution trends may only be speculated at this time, it is highly evident that smaller interparticle distances cause profound interactions which significantly impact oxidation, reduction and dissolution.

With larger particles size of $6.8 \mathrm{~nm}$ compared to $1.9 \mathrm{~nm}$, the Gibbs-Thomson equation dictates a more positive oxidation potential for larger particles, ${ }^{36-38}$ and this system is less easily passivated than the 3D system. ${ }^{48,49}$ This explains the distinctly different shapes of the dissolution rate profiles during the ADT: the $2 \mathrm{D}$ systems fit a more linear trend. However, the recorded mass-specific reduction charge at $0.05 \mathrm{~V}_{\mathrm{RHE}}$ after the ADT follows a similar slope to the 3D system of smaller particle size. As particles shrink during the ADT, they will become more easily passivated due to mechanism (3). This is further corroborated by the extended ADT to 2500 cycles (Fig. S17), where the dissolution rate ceases as shrinking particles become heavily passivated, and the reduction charge after 1000 or 2500 cycles becomes identical.

The study presented here investigated fundamental aspects of PEMFC catalyst degradation, while some important aspects of applied systems should be considered. In real PEMFC devices, the effect of ionomer and interaction with Pt influences performance and degradation. ${ }^{1,2}$ However, in situ/online dissolution studies as conducted here are currently infeasible on a full cell level, and a bottomup approach gives us valuable insights that can predict PEMFC degradation. For instance, Pt dissolution in full cell MEAs is known to be most pronounced close to the membrane, resulting in a $\mathrm{Pt}$ "depletion band," where a significant portion of the Pt is redeposited within the membrane itself. ${ }^{50}$ This is not unexpected, as dissolved $\mathrm{Pt}^{\mathrm{n}+}$ atoms require a conductive medium (ionomer/membrane), and effectively increased degrees of freedom close to the membrane allow increased $\mathrm{Pt}^{\mathrm{n}+}$ diffusion. Therefore, decreasing Pt interparticle distance ( $\mathrm{Pt}$ density) near the membrane should alleviate the depletion band effect, as was found by $\mathrm{Yu}$ et al. ${ }^{50}$

'An interesting study of Lopes et al. has demonstrated the effective use of Pt-Au core-shell/adlayer structures to improve catalyst stability towards degradation, ${ }^{51}$ a technique known to hinder Pt dissolution. ${ }^{52,53} \mathrm{As}$ is common place in rotating disk electrode activity studies, the Pt loading, i.e. the mass of Pt on the electrode, was kept constant. Therefore, although experiments of this work suggest that increasing interparticle distance decreases area-specific Pt dissolution, the thickness of the catalyst layer was drastically changed between electrodes, possibly up to a factor of four, skewing firm conclusions on the impact of interparticle distance. The large impact of catalyst thickness was previously studied by Keeley et al., ${ }^{13}$ which can account for this seemingly contradictory observation. Such a comparison highlights the need for investigations which independently vary parameters such as interparticle distance and particle size, which was not the focus of Lopes et al.

Current applied PEMFC electocatalysts take advantage of the high activity of Pt-transition metal alloys in vehicles such as the Toyota Mirai. ${ }^{54}$ The dissolution of the alloying metal will also contribute to degradation, via loss of activity enhancement. ${ }^{55-57}$ Dissolution of the alloying metal can cause an increased particle surface area and enhance dissolution of $\mathrm{Pt}$, while Pt dissolution itself can expose additional alloy towards further dissolution. ${ }^{58-61}$ Therefore, increased Pt dissolution remains detrimental to alloyed systems. However, the composition, nature of the alloying metal, initial leaching procedure and applied potential protocol (i.e. accelerated durability test) all drastically impact the dissolution of both metals. Furthermore, inhomogenieties in the support material have been shown to impact $\mathrm{Pt}$ dissolution, emphasizing possible influences of the carbon structure. ${ }^{62}$ Here, the single, fundamental parameter of interparticle distance for pure Pt nanoparticles was varied independently of others, while additional studies will be 
required to definitively determine the impacts of additional variables in increasingly complex catalytic systems.

In addition to the complexity of alloyed systems, the complete degradation of PEMFC catalysts is convoluted. The additional degradation mechanisms of carbon corrosion, Ostwald ripening, coalescence and particle detachment are all intricately intertwined with Pt dissolution. ${ }^{36}$ Following the discoveries presented here on the impact of interparticle distance on Pt dissolution, future studies should investigate the impact on the other detrimental degradation mechanisms.

Nevertheless, the observations on both 2D model systems and 3D porous $\mathrm{Pt} /$ Vulcan materials clearly demonstrate a dissolution dependence on interparticle distance, governed by three different mechanisms. Mechanism (3) contributes to this dependence by altering the redox behavior of the nanoparticles, which can be further compounded by the Gibbs-Thomson (particle-size) effect as nanoparticles shrink from electrochemical dissolution. Therefore, in conventional half-cell ADT experiments, potential limits should be chosen carefully with regards to the large variety of loadings and particle sizes in experimental and commercially available electrocatalytic materials.

It is also important for future engineers to take such considerations into account when designing PEMFC stacks in order to optimize performance and lifetime. Very high loadings can lead to nanoparticle agglomeration and lower Pt utilization, while very low loadings may cause increased mass transport, both lowering performance. As Pt dissolution decreases with increased loading, the clear goal would be to synthesize $\mathrm{Pt} / \mathrm{C}$ materials with as high loadings as possible without substantial agglomeration effects. In the quest for increasingly active ORR electrocatalysts to reduce required $\mathrm{Pt}$ quantities in PEMFCs, from this perspective lower loadings should come in the form of thin catalyst layers made from high wt.\% Pt/C materials. However, thin catalyst layers may present their own performance and degradation issues. ${ }^{1,2,63-70}$ Furthermore, the effect of interparticle distance on $\mathrm{Pt}$ dissolution and catalyst layer degradation in full cell MEAs still remains to be explored.

\section{Conclusions}

A clear coverage/loading effect on transient Pt electrochemical dissolution has been observed for model 2D mass-selected systems and applied 3D Pt/Vulcan materials. With increased coverage/ loading mass-specific electrochemical dissolution significantly decreases and stability increases. These observations may be attributed to three possible mechanisms: (1) increased local $\mathrm{Pt}^{\mathrm{n}+}$ ion concentrations causing a shift in the Nernst equilibrium potential for dissolution, (2) increased probability for $\mathrm{Pt}^{\mathrm{n}+}$ ion redeposition, (3) increasing overlap between the nanoparticles electric double layer and coupling/with pronounced dipole-dipole interactions between neighboring nanoparticles. Further ex situ characterization has provided evidence of (3) where Pt oxidation decreases in XPS spectra, and EXAFS data unveils decreasing Pt coordination to oxygen with increasing loading. The consequential passivation effect during accelerated degradation testing is seen to be pronounced for large interparticle distances due to (3), which is diminished for larger particle sizes, emphasizing the importance of potential limits in accelerated degradation test experiments with varied loading or particle size.

\section{Acknowledgments}

This work was funded by the Villum Foundation V-SUSTAIN grant 9455 to the Villum Center for the Science of Sustainable Fuels and Chemicals and the Swiss National Science Foundation (SNSF) via the project No. 200021_184742. The Escalab 250 Xi XPS facility has been co-funded by the Deutsche Forschungsgemeinschaft (INST 184/ 144-1 FUGG). Bundesministerium für Bildung und Forschung (BMBF, FKZ 03SF0539) are gratefully acknowledged. The SLS is acknowledged for the provision of beamtime at the SuperXAS beamline (project ID 20161303). Diamond Light Source, Hartwell,
UK is thanked-in particular Dr. Giannantonio Cibin and AnnKathrin Geiger-for access to synchrotron beamline B18 (proposal SP12746). We thank Dr. L. Theil Kuhn and Dr. S. B. Simonsen, Technical University of Denmark for access to TEM facilities. The access to the microscope for IL-TEM imaging was made possible through the School for Micro- and Nanostructure \& Center for Nanoanalysis and Electron Microscopy (CENEM) at the FriedrichAlexander-University Erlangen-Nürnberg.

\section{Experimental}

Full experimental procedures can be found in the first section of the associated supporting information.

\section{ORCID}

Daniel J. S. Sandbeck (D) https://orcid.org/0000-0002-7618-3574 Niklas Mørch Secher (D) https://orcid.org/0000-0003-1027-5027 Masanori Inaba (iD https://orcid.org/0000-0002-2292-7961 Jonathan Quinson (iD https://orcid.org/0000-0002-9374-9330 Jakob Ejler Sørensen (D) https://orcid.org/0000-0001-8834-1616 Jakob Kibsgaard (iD https://orcid.org/0000-0002-9219-816X Alessandro Zana (iD https://orcid.org/0000-0003-0588-3116 Francesco Bizzotto (iD https://orcid.org/0000-0002-5624-6697 Florian D. Speck (iD https://orcid.org/0000-0002-7649-9261 Michael T. Y. Paul (iD https://orcid.org/0000-0003-2206-0592 Alexandra Dworzak (iD https://orcid.org/0000-0002-8689-9517 Carsten Dosche (iD https://orcid.org/0000-0003-3837-5169 Mehtap Oezaslan (iD https://orcid.org/0000-0001-8545-7576 Ib Chorkendorff (iD https://orcid.org/0000-0003-2738-0325 Matthias Arenz (iD https://orcid.org/0000-0001-9765-4315 Serhiy Cherevko httpsi//orcid.org/0000-0002-7188-4857

\section{References}

1. A. Kongkanand and M. F. Mathias, J. Phys. Chem. Lett., 7, 1127 (2016).

2. P. Gazdzicki, J. Mitzel, A. M. Dreizler, M. Schulze, and K. A. Friedrich, Fuel Cells, 18, 270 (2018)

3. V. R. Stamenkovic, B. Fowler, B. S. Mun, G. Wang, P. N. Ross, C. A. Lucas, and N. M. Marković, Science, 315, 493 (2007).

4. P. Hernandez-Fernandez et al., Nat. Chem., 6, 732 (2014).

5. M. Escudero-Escribano, P. Malacrida, M. H. Hansen, U. G. Vej-Hansen, A. Velázquez-Palenzuela, V. Tripkovic, J. Schiøtz, J. Rossmeisl, I. E. L. Stephens, and I. Chorkendorff, Science, 352, 73 (2016).

6. D. Li et al., Energy Environ. Sci., 7, 4061 (2014).

7. M. Oezaslan, F. Hasché, and P. Strasser, J. Phys. Chem. Lett., 4, 3273 (2013).

8. I. A. Safo, C. Dosche, and M. Oezaslan, ChemPhysChem, 20, 15 (2019).

9. S. Chen, H. A. Gasteiger, K. Hayakawa, T. Tada, and Y. Shao-Horn, J. Electrochem. Soc., 157, A82 (2010)

10. C. E. Carlton, S. Chen, P. J. Ferreira, L. F. Allard, and Y. Shao-Horn, J. Phys. Chem. Lett., 3, 161 (2012).

11. S. Cherevko, Curr. Opin. Electrochem., 8, 118 (2018)

12. S. Cherevko, N. Kulyk, and K. J. J. Mayrhofer, Nano Energy, 29, 275 (2016).

13. G. P. Keeley, S. Cherevko, and K. J. Mayrhofer, ChemElectroChem, 3, 51 (2016).

14. J. Speder, I. Spanos, A. Zana, J. J. K. Kirkensgaard, K. Mortensen, L. Altmann, M. Bäumer, and M. Arenz, Surf. Sci., 631, 278 (2015).

15. J. Speder, L. Altmann, M. Bäumer, J. J. K. Kirkensgaard, K. Mortensen, and M. Arenz, RSC Adv., 4, 14971 (2014).

16. E. Fabbri, S. Taylor, A. Rabis, P. Levecque, O. Conrad, R. Kötz, and T. J. Schmidt, ChemCatChem, 6, 1410 (2014).

17. M. Nesselberger et al., Nat. Mater., 12, 919 (2013)

18. M. Inaba, "Systematic study of Pt/C catalysts for polymer electrolyte membrane fuel cells." Department of Chemistry (Unversity of Copenhagen, Copenhagen, Denmark) (2018).

19. S. Taylor, E. Fabbri, P. Levecque, T. J. Schmidt, and O. Conrad, Electrocatalysis, 7, 287 (2016).

20. J. Quinson et al., ACS Catal., 8, 6627 (2018).

21. J. Speder, L. Altmann, M. Roefzaad, M. Baumer, J. J. Kirkensgaard, K. Mortensen, and M. Arenz, Phys. Chem. Chem. Phys., 15, 3602 (2013).

22. S. Proch, K. Kodama, M. Inaba, K. Oishi, N. Takahashi, and Y. Morimoto, Electrocatalysis, 7, 249 (2016).

23. J. Speder, A. Zana, I. Spanos, J. J. K. Kirkensgaard, K. Mortensen, and M. Arenz, Electrochem. Commun., 34, 153 (2013).

24. J. Speder, A. Zana, I. Spanos, J. J. K. Kirkensgaard, K. Mortensen, M. Hanzlik, and M. Arenz, J. Power Sources, 261, 14 (2014).

25. E. D. Goodman, A. C. Johnston-Peck, E. M. Dietze, C. J. Wrasman, A. S. Hoffman, F. Abild-Pedersen, S. R. Bare, P. N. Plessow, and M. Cargnello, Nat. Catal., 2, 748 (2019). 
26. G. S. Harzer, A. Orfanidi, H. El-Sayed, P. Madkikar, and H. A. Gasteiger, J. Electrochem. Soc., 165, F770 (2018).

27. Y. Garsany, O. A. Baturina, K. E. Swider-Lyons, and S. S. Kocha, Anal. Chem., 82, $6321(2010)$.

28. Y. Garsany, I. L. Singer, and K. E. Swider-Lyons, J. Electroanal. Chem., 662, 396 (2011).

29. Y. Garsany, J. Ge, J. St-Pierre, R. Rocheleau, and K. E. Swider-Lyons, J. Electrochem. Soc., 161, F628 (2014).

30. K. Shinozaki, J. W. Zack, S. Pylypenko, B. S. Pivovar, and S. S. Kocha, J. Electrochem. Soc., 162, F1384 (2015).

31. M. Inaba, J. Quinson, and M. Arenz, J. Power Sources, 353, 19 (2017).

32. M. Inaba, J. Quinson, J. R. Bucher, and M. Arenz, J. Vis. Exp., 133, 10 (2018).

33. K. Ke, K. Hiroshima, Y. Kamitaka, T. Hatanaka, and Y. Morimoto, Electrochim. Acta, 72, 120 (2012)

34. R. Sharma and S. M. Andersen, ACS Appl. Mater. Interfaces, 10, 38125 (2018).

35. E. Pizzutilo, S. Geiger, J. P. Grote, A. Mingers, K. J. J. Mayrhofer, M. Arenz, and S. Cherevko, J. Electrochem. Soc., 163, F1510 (2016).

36. J. C. Meier, C. Galeano, I. Katsounaros, J. Witte, H. J. Bongard, A. A. Topalov, C. Baldizzone, S. Mezzavilla, F. Schuth, and K. J. J. Mayrhofer, Beilstein J. Nanotechnol., 5, 44 (2014).

37. W. J. Plieth, J. Phys. Chem., 86, 3166 (1982).

38. K. J. Mayrhofer, B. B. Blizanac, M. Arenz, V. R. Stamenkovic, P. N. Ross, and N. M. Markovic, J. Phys. Chem. B, 109, 14433 (2005).

39. K. Yu, D. J. Groom, X. Wang, Z. Yang, M. Gummalla, S. C. Ball, D. J. Myers, and P. J. Ferreira, Chem. Mater., 26, 5540 (2014).

40. L. Tang, B. Han, K. Persson, C. Friesen, T. He, K. Sieradzki, and G. Ceder, J. Am. Chem. Soc., 132, 596 (2010).

41. L. Tang, X. Li, R. C. Cammarata, C. Friesen, and K. Sieradzki, J. Am. Chem. Soc., 132, 11722 (2010).

42. J. Huang, J. Zhang, and M. H. Eikerling, J. Phys. Chem. C, 121, 4806 (2017).

43. C. B. Murray, C. R. Kagan, and M. G. Bawendi, Annu. Rev. Mater. Sci., 30, 545 (2000).

44. H. Liu, B. S. Mun, G. Thornton, S. R. Isaacs, Y.-S. Shon, D. F. Ogletree, and M. Salmeron, Phys. Rev. B, 72, 155430 (2005).

45. J. Liao, S. Blok, S. J. van der Molen, S. Diefenbach, A. W. Holleitner, C. Schonenberger, A. Vladyka, and M. Calame, Chem. Soc. Rev., 44, 999 (2015).

46. D. V. Talapin, J. S. Lee, M. V. Kovalenko, and E. V. Shevchenko, Chem. Rev., 110 389 (2010)

47. J. Ustarroz, I. M. Ornelas, G. Zhang, D. Perry, M. Kang, C. L. Bentley, M. Walker, and P. R. Unwin, ACS Catal., 8, 6775 (2018).
48. D. J. S. Sandbeck, N. M. Secher, F. D. Speck, J. E. Sørensen, J. Kibsgaard, I. Chorkendorff, and S. Cherevko, ACS Catal., 10, 6281 (2020).

49. D. J. S. Sandbeck, M. Inaba, J. Quinson, J. Bucher, A. Zana, M. Arenz, and S. Cherevko, ACS Appl. Mater. Interfaces, 12, 25718 (2020).

50. H. Yu, A. Baricci, A. Casalegno, L. Guetaz, L. Bonville, and R. Maric, Electrochim. Acta, 247, 1169 (2017).

51. P. P. Lopes et al., Nat. Mater., 19, 1207 (2020).

52. S. Cherevko, G. P. Keeley, N. Kulyk, and K. J. J. Mayrhofer, J. Electrochem. Soc., 163, I1228 (2016).

53. J. Zhang, K. Sasaki, E. Sutter, and R. R. Adzic, Science, 315, 220 (2007).

54. R. L. Borup, R. Mukundan, K. More, K. C. Neyerlin, A. Z. Weber, D. Myers, and R. Ahluwalia, 233rd Meeting of the Electrochemical Society, r. M. o. t. E. Society Editor, Seatle, Washington, USA (2018).

55. I. Spanos, K. Dideriksen, J. J. Kirkensgaard, S. Jelavic, and M. Arenz, Phys. Chem. Chem. Phys., 17, 28044 (2015).

56. V. Beermann, M. E. Holtz, E. Padgett, J. F. de Araujo, D. A. Muller, and P. Strasser, Energy Environ. Sci., 12, 2476 (2019).

57. Q. Jia et al., Nano Lett., 18, 798 (2018).

58. P. Jovanovič et al., J. Power Sources, 327, 675 (2016).

59. M. Gatalo, P. Jovanovič, U. Petek, M. Šala, V. S. Šelih, F. Ruiz-Zepeda, M. Bele, N. Hodnik, and M. Gaberšček, ACS Appl. Energy Mater., 2, 3131 (2019).

60. R. K. Ahluwalia, D. D. Papadias, N. N. Kariuki, J.-K. Peng, X. Wang, Y. Tsai, D. G. Graczyk, and D. J. Myers, J. Electrochem. Soc., 165, F3024 (2018).

61. C. Baldizzone, L. Gan, N. Hodnik, G. P. Keeley, A. Kostka, M. Heggen, P. Strasser, and K. J. J. Mayrhofer, ACS Catal., 5, 5000 (2015).

62. P. Jovanovic, U. Petek, N. Hodnik, F. Ruiz-Zepeda, M. Gatalo, M. Sala, V. S. Selih, T. P. Fellinger, and M. Gaberscek, Phys. Chem. Chem. Phys., 19, 21446 (2017).

63. M. K. Debe, A. K. Schmoeckel, S. M. Hendricks, G. D. Vernstrom, G. M. Haugen, and R. T. Atanasoki, ECS Trans., 1, 51 (2006).

64. A. J. Steinbach, K. Noda, and M. K. Debe, ECS Trans., 1, 835 (2006).

65. M. K. Debe, A. K. Schmoeckel, G. D. Vernstrom, and R. Atanasoski, J. Power Sources, 161, 1002 (2006).

66. A. Bonakdarpour, K. Stevens, G. D. Vernstrom, R. Atanasoski, A. K. Schmoeckel, M. K. Debe, and J. R. Dahn, Electrochim. Acta, 53, 688 (2007).

67. P. K. Sinha, W. Gu, A. Kongkanand, and E. Thompson, J. Electrochem. Soc., 158 B831 (2011).

68. A. Kongkanand, M. Dioguardi, C. Ji, and E. L. Thompson, J. Electrochem. Soc., 159, F405 (2012)

69. A. Kongkanand, J. Zhang, Z. Liu, Y.-H. Lai, P. Sinha, E. L. Thompson, and R. Makharia, J. Electrochem. Soc., 161, F744 (2014).

70. A. J. Steinbach et al., ECS Trans., 80, 659 (2017). 